\title{
Erratum to: Professional and Ethical Challenges in Determinations of Causality of Psychological Disability
}

\author{
Shadi Gholizadeh $^{1}$ - Vanessa L. Malcarne ${ }^{1,2}$
}

Published online: 23 January 2016

(C) Springer Science+Business Media New York 2016

Erratum to: Psychol. Inj. and Law (2015) 8(4):334-347

DOI 10.1007/s12207-015-9237-z

The original version of this article unfortunately contained the following errors.

On page 336 , the quotation, “...like the yeti, sought after, largely unseen" (Harris, 2012), is missing the word 'but' and has an additional comma.

- The correction sentence is: Far from a straightforward aspiration, properly providing substantial evidence in medical legal reports has been described as, “... like the yeti, sought after but largely unseen" (Harris, 2012).

On page 340 and 341, the three references to Harris (2012) should be for McGavin (2005).

- On page 340 on line 4, "Harris (2012) unpacked the complexities..." should be attributed to McGavin (2005). The correct sentence should be:

McGavin (2005) unpacked the complexities associated with presenting "substantial evidence" and commented upon the "slippery slope" with which QMEs are now faced in relation to preexisting conditions and vulnerabilities.

- On page 341, "Giving the example of an outdoor worker with red hair and freckles who develops melanoma, Harris

The online version of the original article can be found at http://dx.doi.org/ 10.1007/s12207-015-9237-z.

Shadi Gholizadeh

sgholiza@ucsd.edu

1 SDSU/UC San Diego Joint Doctoral Program in Clinical Psychology, 6363 Alvarado Court, Suite 103, San Diego, CA 92120-4913, USA

2 Department of Psychology, San Diego State University, San Diego, CA, USA gave the extreme example" should be attributed to McGavin (2005). The correct sentence should be "Giving the example of an outdoor worker with red hair and freckles who develops melanoma, McGavin gave the extreme example of a QME apportioning the cancer to the genetic predisposition.

- On page 341, "To adapt an example, Harris (2012) provided in her analysis that if we consider a worker..." should be attributed to McGavin (2005) and 'her' should be changed to 'his'. The correct sentence should be: To adapt an example, McGavin (2005) provided in his analysis that if we consider a worker with a preexisting diagnosis of schizophrenia who is alleging MDD resulting from working in a hostile work environment, key questions that would need to be answered in the evaluative report would be, "Are you comparing the worker to someone who had the same experiences and treatment but did not have schizophrenia?"

- The following reference should be added to the reference list between Matsumoto A. (1994) and Otto, R. K., \&Heilbrun, K. (2002):

McGavin, Y. (2005).Escobedo and the slippery slope of irrelevancy. WorkCompCentral. [Web post]. Retrieved August 01, 2015, fromhttps://www.workcompcentral.com/columns/ show/id/u128d05h600742wx4f7jos 